\title{
Quality Education of Political Thought in Mathematical Modeling
}

\author{
Zheng Gao* \\ Chengdu No.7 High school \\ Chengdu, Sichuan, P.R. China \\ Email: 303081664@qq.com \\ *Corresponding author
}

\author{
Xiaojing Tao \\ Weihe Primary school \\ Zhengzhou, Henan, P.R. China \\ Email:1032255802@qq.com
}

\author{
Yubin Pan \\ School of Mathematics Science \\ University of Electronic Science and Technology of China \\ Chengdu, P.R. China \\ Email: yubinpan2014@163.com
}

\begin{abstract}
Mathematical modeling provides students with rich and real materials for patriotism and national conditions education, and it is also an effective way to publicize national policies and an important means to cultivate students' dialectical materialist worldview. In addition, mathematical modeling has the function of cultivating students' rigorous academic attitude and advanced scientific and technological consciousness. The mathematical beauty shown in the modeling has created conditions for the preliminary formation of students' mathematical aesthetic concept. This paper mainly introduces how to cultivate students' political and ideological quality through mathematical modeling.
\end{abstract}

Keywords-mathematical modeling; ideology political; education research

\section{INTRODUCTION}

Mathematical modeling is the mathematical abstraction of practical problems, expressing problems in mathematical language, constructing models by mathematical methods to solve practical problems, including finding problems from the perspective of mathematics in the actual situation, putting forward problems, analyzing problems, establishing models, determining parameters, calculating and solving, testing and perfecting models, and finally solving practical problems. The mathematical model builds a bridge between mathematics and the outside world, which is an important form of mathematical application. Mathematical modeling is the basic means to solve practical problems by using mathematics, and it is also the driving force to promote the development of mathematics[1].

Through the study of mathematical modeling course, first of all, students can consciously express the real world in mathematical language, discover and ask problems, and understand the relationship between mathematics and reality. Secondly, students learn to solve practical problems with mathematical models and accumulate experience in mathematical practice. Finally, students should understand the role of the mathematical model in many fields of science, society and engineering technology, so as to stimulate the students' practical ability, enhance the consciousness of innovation and the scientific spirit[2,3]. This is not only the need for the development of mathematics and the improvement of students' mathematical quality, but also the inevitable requirement of social development. Many countries regard mathematical modeling as an important subject for research, and there are many knowledgeable articles on the theory and method of mathematical modeling[4]. However, we have not paid enough attention to how to give full play to the ideological and political education function of mathematical modeling, to create a good situation for the education of students' ideological quality, to cultivate the dialectical materialist worldview and to format the aesthetic concept, so it is necessary to discuss this problem.

\section{Mathematical Modeling PROVIDES Rich AND REAL MATERIALS FOR PATRIOTISM AND NATIONAL CONDITION EDUCATION}

Patriotism is the most cohesive and emotional appeal flag. In order to make students love their country, first of all, they should understand the history of their country. Mathematical modeling is an important way for students to understand the mathematical history of the country. "Problem of Chicken and rabbit in the same cage", "problem of a hundred chickens" in The Nine Chapters on the Mathematical Art and "Sun Zi's problem" in Sun zi suan jing, these simple number theory with application problems have important status in the history of mathematics, and it contains concise, concrete and characteristic mathematical principles. The problems studied in The Nine Chapters on the Mathematical Art by Qin Jiushao, an ancient Chinese mathematician, highlight the practicability and are drawn from the categories of social economy, culture, science and technology[5]. He went deep into the civil and social reality, reflected the problems of social life as mathematical problems, and was good at expressing boring mathematics in interesting strokes. His works are examples of mathematics. In particular, these typical problems, such as "wang shan yuan jin", "dou an ce shui" and "tian xia di yi an", 
have a great influence on the history of mathematics, and their inherent significance has gone far beyond the limited world of pure mathematics, and the mathematical thought provided for future generations is even more highly valuable inspiration.

If we promote the great contribution and far-reaching influence of these "most wonderful mathematical inventions" to the world culture through mathematical modeling, we can enhance students' national self-esteem and pride, enhance their sense of urgency of the times, correct their lack of ambition and blindly inherit the previous thoughts

Linking practical problems with mathematics and designing mathematical modeling problems can deepen students' understanding of real national conditions. For example, the mathematical modelings based on population growth, land use, grain and resources as the background, requires students to flexibly use equations, inequalities, binomial expansion and approximate operation to accurately express the relationship among cultivated land, population and grain possession, which can push students' application consciousness to the height of thinking about the development of the country in the future century, and give students a vivid national condition education class. It can be seen that mathematical modeling is rich in the spirit of patriotism and state-of-the-art education, which is more data-sensitive and more realistic than the other materials.

\section{MATHEMATICAL MOdELING Is AN EFFECTIVE WAY TO PROPAGATE NATIONAL POLICY}

On the occasion of the reform of the country, the change of people's living standards, the prospect of reform can be reflected in the problem of mathematical modeling. Though the teaching of these modeling problems, students can learn national policies, understand and care about social problems. For example, the problems based on the countryside, through real people and real events, by using mathematical modeling to calculate farmer's income, the advantages of rural policies are irrefutably proved with scientific data, which can strengthen the students' trust in the policy of the ruling party. Again, "Government Grants", "Price Controls", "Market Price”, "Equilibrium Price", these economic nouns, which come from real problems, permeates the breath of life. The student flexible use the knowledge of function, equation, and inequality to study the amount of government subsidy, which can make them understand that our country has both planned economy and market economy. Some enterprises have to be supported, while others are regulated by the market, so it is impossible for our country to have hyperinflation.

At present, the market economy is the main melody of society politics and economic life, and the student are the activists among them. The market economy needs the student to master more and more useful mathematical knowledge, to master all sorts of mathematical methods to solve problems in the market economy, and to have a certain degree of modeling ability, which is the inevitable requirement of the society. With the continue improvement of the market economy and the further advance of the contract system, stock system and renting and leasing system, each producer is also an operator, the quality of product, marketing and personal interests will be directly linked. Therefore, a series of problems, such as input, output, loan, benefit, stock, market prediction, risk assessment and son, will become the most common phenomenon in people's social life. Economical activities in people's daily life are colorful, a series of economic activities, such as buy and sell, deposit and insurance, stock and bond and so on, requires people to have an economic mind and a sense of merchandise, all of which are closely related with mathematical modeling. For example, interest and rate, statistics and probability, operation and optimization and analysis and decision-making, the student in social-economic life have become familiar with these problems into mathematical problems, which can not only improve the interest in solving mathematics problems, but also promote students to solve the most practical problems in current daily life and production, so as to make them understand the society, care about the reform of economic system, and participate in the wave of national market economy and make due contribution to the prosperity and stability of the country.

\section{MATHEMATICAL MODELING Is AN IMPORTANT MEANS to Cultivate Students' Dialectical MATERIALIST WORLDVIEW}

Mathematics modeling is to use mathematics to solve practical problems, to use mathematical language and method to describe the actual problems. These mathematical expressions are mathematics models. For the established mathematics models, we need to use some specific techniques (such as reasoning proof, calculation, etc.) to solve them, and verify them with the actual situation, so as to achieve the purpose of solving actual problems. From the essence of the mathematical modeling, it shows two leaps of the dialectical process of cognition. The first leap is to generate the perceptual knowledge from the practical problems, and then develop to rational knowledge by using mathematical knowledge, finally establish mathematical modeling. The second leap is to scientifically verify the conclusion and to guild practice, which is the process from theory to practise. For example, with the help of mathematical modeling of the varying of concentration of a drug with time and space in human's body, medical experts can analyze the drug's benefit, so as to effectively guide clinical use of the drug. Factory directors and mangers plan mathematical modeling for the reasonable arrangement of production and sale, which can be used to guild practice and obtain higher economic benefits.

Mathematical modeling can impel students to transform from directness and concreteness of perceptual cognition to indirectness and abstractness of rational cognition, which can reveal the dialectical process of human cognition that condensed in the process of mathematical modeling. Abstract the mathematical model from practice, in order to reveal the essence of objective things more deeply and generally, the abstractness of mathematics brings the universality of application, and embodies the dialectical process of cognition.

Mathematics is the reflection of the real world in human's brain. Real-world is full of contradiction, so mathematics is bound to be full of contradiction. The practical problems in actual life are the concentrated reflection of contradictions, and there are also contradictory concentrated manifestations in mathematical modeling. Known and unknown, constant and variable, finite and infinite, equal and unequal are all directly 
manifested in the form of unity and opposites, showing the contradiction of modeling problems. This shows that mathematical modeling provides a broad world for cultivating the dialectics of thinking. The key of mathematical modeling is to find the main contradiction of things, and then we can consciously understand the essence from the contradiction among these things, so as to conquer the one-sidedness and absolute of thinking. This is very beneficial to the students to face up to the contradiction, to expose the contradiction and to solve the contradiction in the face of the complication things.

\section{Mathematical Modeling Has the Function of}

CULTIVATING STUDENTS’ RIgOROUS ACADEMIC ATTITUDE AND ADVANCED SCIENTIFIC AND TECHNOLOGICAL

\section{CONSCIOUSNESS}

Mathematical modeling is to use the operational properties, such as rules, formulas, truths, theorem, corollary and so on in mathematics to extract practical problems into mathematical expressions and then solve them. They all have logical preciseness. Combined with this characteristic, mathematical modeling teaching can play a role in cultivating students' rigorous scientific attitude and good learning habits.

Modeling ability is the solver's comprehensive application of all kinds of abilities, involving literacy understanding ability, familiarity with practical problems, mastery of relevant knowledge, good psychological quality, innovative spirit and creative ability, as well as the comprehensive application of various scientific thinking methods, such as observation, analysis, comparison, comprehension, generalization etc., so that students can develop a good mathematical cognitive structure and grasp the train of thought, skill and method of solving practical problems in mathematics, and them effectively improve students' mathematical modeling and application skills, stimulate students to learn mathematics enthusiasm, cultivate their courage to climb the peak of science.

At present, the three gorges hydroelectric power station is the biggest hydro-project in the world, involving a series of problems, such as power generation, flood control, shipping, environment protect, breeding, immigration, preservation of cultural relics, war defence, etc. The complicated of the project can be imagined. From the closure Yangtze river to the final generation of electricity, the arrangement and planning of this series of projects are transformed into mathematical problems through mathematical modeling. The large computer is used to carry out the operation for two years, and some other schemes are put forward for analysis and comparison. Finally, the construction scheme is worked out. This sufficiently shows the importance of mathematical modeling. Because mathematical modeling contains rich science and technology knowledge, it is bound to have a subtle effect, which can promote students to love science, learn science, use science, to have advanced scientific and technological consciousness, and to strengthen their understanding that "science and technology is the primary productive force".

\section{Mathematical BeAuty in Mathematical} MODELING CREATES CONDITIONS FOR THE PRELIMINARY FORMATION OF THE AESTHETIC CONCEPT OF THE EDUCATED

Mathematical beauty in mathematical modeling contains abundant contents, such as the beauty of rigorous and refine language in describing problems, the symmetrical beauty of geometric form and mathematical formula, the science beauty in revealing quantitative relations, the singular beauty of mathematics model solution, and harmonious beauty in the whole process of mathematical modeling all show the infinite charm contained in the aesthetic object of mathematical modeling. If we show students the elegant form of practical problems, they are bound to be influenced by beauty and experience a certain degree of pleasure, thus germinating the concept of beauty in the depths of their hearts.

The complexity of real-life determines the diversity of the mathematical models that reflect these problems. In a sense, different mathematical modelings for one problem is the specific application of aesthetic consciousness, which can expand new ideas, stimulate students to explore unknowns. Looking for the best mathematical modeling can cultivate students to unremitting pursuit of beauty.

The beauty of applying mathematics is an important aspect of mathematics beauty, which shows the perfection and harmony of mathematics to the outside world. Different people apply same mathematical concepts and methods to study different things, and different things are subject to the same mathematical law, which fully reflects the beauty of applying mathematics in mathematical modeling.

The mathematical modeling has the characteristics of large information, high flexibility, and strong pioneering nature. If the teacher can take full advantage of the beauty of mathematical modeling, they can set the strings of the student's heart and stimulate the enthusiasm of the beautiful creation.

\section{CONCLUSION}

Mathematical modeling, with its rich and colorful content, has some favorable factors for the implementation of ideological and political education. At the same time, because the whole process of mathematical modeling is a real mathematical activity, there is no trace of boring preaching, compared with other ideological and political education, it has the characteristics of "nourishing all things silently", students must be easier to accept, and the effect of education is selfevident. In that case, the ideological and political quality education in mathematical modeling should be brought into full play and the perfect unity of teaching and education should be realized, which should be attached great importance to by the majority of mathematics teachers.

\section{REFERENCES}

[1] Mathematics Curriculum Standards for Senior High Schools (2017 Edition).(In Chinese)

[2] J. Fu, H. Zhu, X.C. Wang. Practice and thinking on cultivating students' innovative ability in Mathematical Modeling Teaching [J]. Journal of Mathematics Education, 2007,16 (4): 93- 95.(In Chinese) 
[3] J. Pu, C.L. Zhang, S.C. Li. Exploring mathematical modeling teaching reform and improving college students' comprehensive quality[J]. Chinese University Teaching, 2011(12):24-25.(In Chinese)
[4] Z.S.Cai.Mathematical modeling: principle and method [M]. China Ocean Press, 2000.(In Chinese).

[5] S.C. Guo, The translation and annotation of jiu zhang suan shu. Shanghai Classics Publishing House 2009.(In Chinese) 